\title{
Recent Advances on Mathematical Modeling and Control Methods for Complex Vehicle Systems
}

\author{
Hamid Reza Karimi, ${ }^{1}$ Zheping Yan, ${ }^{2}$ Yuxin Zhao, ${ }^{2}$ Weichao Sun, ${ }^{3}$ and Mohammed Chadli ${ }^{4}$ \\ ${ }^{1}$ Department of Engineering, Faculty of Engineering and Science, University of Agder, 4898 Grimstad, Norway \\ ${ }^{2}$ College of Automation, Harbin Engineering University, Nantong Street \#145, Nangang District, Harbin, China \\ ${ }^{3}$ School of Astronautics, Harbin Institute of Technology, P.O. Box 3015, Yikuang Street \#2, Nangang District, Harbin, China \\ ${ }^{4}$ Laboratory of Modeling, Information \& Systems, University of Picardie Jules Verne, Amiens, France
}

Correspondence should be addressed to Hamid Reza Karimi; hrkarimi@gmail.com

Received 31 October 2014; Accepted 31 October 2014; Published 27 November 2014

Copyright (C) 2014 Hamid Reza Karimi et al. This is an open access article distributed under the Creative Commons Attribution License, which permits unrestricted use, distribution, and reproduction in any medium, provided the original work is properly cited.

Complex vehicle systems such as highway vehicle, surface vehicle, underwater vehicle, space vehicle, and unmanned vehicle play extremely important roles in wide range of industrial sectors like transportation, mineral exploration, and space exploration. Due to the increasing demands on system performance, diversity, and complexity of the tasks, more and more attention has been paid to complex vehicle correlative technologies with full consideration of issues like mathematical modeling, mission planning, robust performance, coordination and formation, optimization, communication, navigation, prognosis, fault diagnosis and isolation, and so on. All aforementioned issues provide a basis for the design and operation of practical complex vehicle systems in order to achieve desired complex tasks. This special issue aims at providing an opportunity for scientists, engineers, and practitioners to propose their latest theoretical and technological achievements in systems control, especially in the aforesaid aspects.

This special issue contains twenty-nine papers, the contents of which are mainly summarized as follows.

"Urban and indoor weak signal tracking using an array tracker with MVA and nonlinear filtering" by J. Ding et al. focuses on the need for weak GPS signal tracking technique, where the tracking loop is unlocked and data bit edge position is unknown. A modified Viterbi algorithm (MVA) based on dynamic programming is developed, and it is applied to GPS bit synchronization to improve bit edge position detection probability. Meanwhile, two combination carrier tracking schemes based on Central Difference Kalman Filter (CDKF) and MVA module are designed for tracking very weak GPS signal. The testing results indicate that the methods can successfully detect bit edge position with high detection probability whether or not the tracking loop is locked.

"Support vector regression-based adaptive divided difference filter for nonlinear state estimation problems" by $\mathrm{H}$. Wang et al. presents a support vector regression-based adaptive divided difference filter (SVRADDF) algorithm for improving the low state estimation accuracy of nonlinear systems, which are typically affected by large initial estimation errors and imprecise prior knowledge of process and measurement noises. The derivative-free SVRADDF algorithm is significantly simpler to compute than other methods and is implemented using only functional evaluations.

"Robust stability clearance of flight control law based on global sensitivity analysis" by L. Ou et al. proposes a new clearance framework based on structural singular value $\mu$ theory and global uncertainty sensitivity analysis (SA) to validate the robust stability of the flight control system of hypersonic flight vehicle, which suffers from a large number of parametrical uncertainties. In this framework, SA serves as the preprocess of uncertain model to be analyzed to help engineers determine which uncertainties affect the stability of the closed loop system more slightly. Instead of analyzing the effect of uncertainties on $\mu$ which involves solving optimal problems repeatedly, a simpler stability analysis function is proposed. Based on this stability analysis function, Sobol 
method, the most widely used global SA method, is extended and applied to the new clearance framework due to its suitability for system with strong nonlinearity and input factors varying in large interval, as well as input factors subjecting to random distributions.

"Probabilistic harmonic calculation in distribution networks with electric vehicle charging stations" by J. Wang et al. provides the integration of a mathematical simulation method for studying the working status of charging stations, which considers influencing factors including random leaving factor, electricity price, and waiting time. Based on the proposed simulation method, the probability distribution of the harmonic currents of EV charging stations is obtained and used in the calculation of the probability harmonic power flow. Then the impacts of EVs and EV charging stations on distribution networks can be analyzed. In case study, the proposed simulation and analysis method is implemented on the IEEE-34 distribution network. The influences of EV arrival rates, the penetration rate, and the accessing location of $\mathrm{EV}$ charging station are also investigated.

In the paper "Sign stability for switched linear systems and its application in flight control" by Q. Wang et al., the sign stability concept in ecological systems is introduced into the analysis and synthesis of switched linear system to explore new control design technique. The necessary and sufficient condition for sign stability of a switched linear system under arbitrary switching is achieved. A new approach for the stabilization of switched system is presented. Although the controllers are devised for each subsystem, respectively, the switched system is sign-stabilized by the constitution of CISSS. The provided method has natural robustness and more design freedoms than the familiar Lyapunov function method, which bears relative conservativeness as the requirement of solving LMIs. The presented technique is validated by an example of flight control within a large-scale flight envelop.

In the paper "An aeromagnetic compensation algorithm for aircraft based on fuzzy adaptive Kalman filter" by Y. Zhang et al., the classical aircraft aeromagnetic compensation algorithm based on Leliak model is analyzed and an aircraft aeromagnetic compensation algorithm based on fuzzy adaptive Kalman filter is proposed, which is a new approach for aircraft to achieve aeromagnetic compensation. Simulation results show that it has better compensation performance without relying on the aircraft attitude.

"Nonlinear dynamical analysis of hydraulic turbine governing systems with nonelastic water hammer effect" by J. Li and Q. Chen proposes a nonlinear mathematical model for hydroturbine governing system (HTGS), and all essential components of HTGS, that is, conduit system, turbine, generator, and hydraulic servosystem, are considered in the model. Using the proposed model, the existence and stability of Hopf bifurcation of an example HTGS are investigated. In addition, chaotic characteristics of the system with different system parameters are studied extensively and presented in the form of bifurcation diagrams, time waveforms, phase space trajectories, Lyapunov exponent, chaotic attractors, and Poincare maps. The simulation results provide a reasonable explanation for the sustained oscillation phenomenon commonly seen in operation of hydroelectric generating set.
"Research on optimal control for the vehicle suspension based on the simulated annealing algorithm" by J. Meng et al. presents a designed method to optimize the weight matrix of the LQR controller by using the simulated annealing algorithm. This method utilizes the random searching characteristics of the algorithm to optimize the weight matrices with the target function of suspension performance indexes. This method improves the design efficiency and control performance of the LQR control and solves the problem of the LQR controller when defining the weight matrices. And a simulation is provided for vehicle active chassis control. The result shows that the active suspension using LQR optimized by the genetic algorithm compared to the chassis controlled by the normal LQR and the passive one shows better performance. Meanwhile, the problem of defining the weight matrices is greatly solved.

"Optimal acceleration-velocity-bounded trajectory planning in dynamic crowd simulation" by F. Yue-wen et al. studies one special type of crowd which is composed of urgent individuals, normal individuals, and normal groups, where three steps are used to construct the crowd simulation in dynamic environment. The first one is that the urgent individuals move forward along a given path around dynamic obstacles and other crowd members. An optimal acceleration-velocitybounded trajectory planning method is utilized to model their behaviors, which ensures that the durations of the generated trajectories are minimal and the urgent individuals are collision-free with dynamic obstacles (e.g., dynamic vehicles). In the second step, a pushing model is adopted to simulate the interactions between urgent members and normal ones, which ensures that the computational cost of the optimal trajectory planning is acceptable. The third step is obligated to imitate the interactions among normal members using collision avoidance behavior and flocking behavior.

Of course, the selected topics and papers are not a comprehensive representation of the area of this special issue. Nonetheless, they represent the rich and many-faceted knowledge that we have the pleasure of sharing with the readers. We hope that readers will find in this special issue not only the published papers on complex vehicle systems but also important questions to be resolved such as mathematical modeling, control strategies, and advanced computational algorithm.

\section{Acknowledgments}

We would like to express appreciation to the authors for their excellent contributions and patience in assisting us. The hard work of all reviewers on these papers is also very greatly acknowledged.

Hamid Reza Karimi Zheping Yan Yuxin Zhao Weichao Sun Mohammed Chadli 


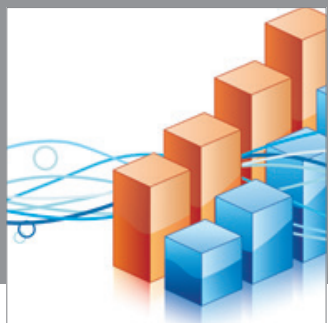

Advances in

Operations Research

mansans

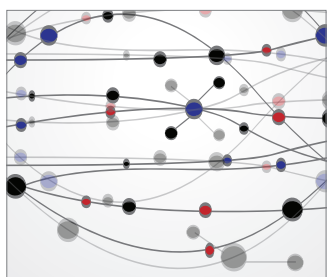

The Scientific World Journal
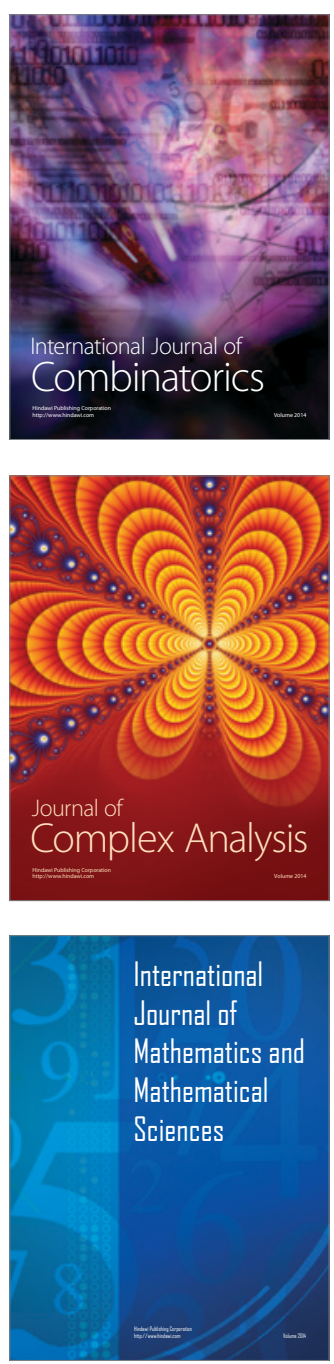
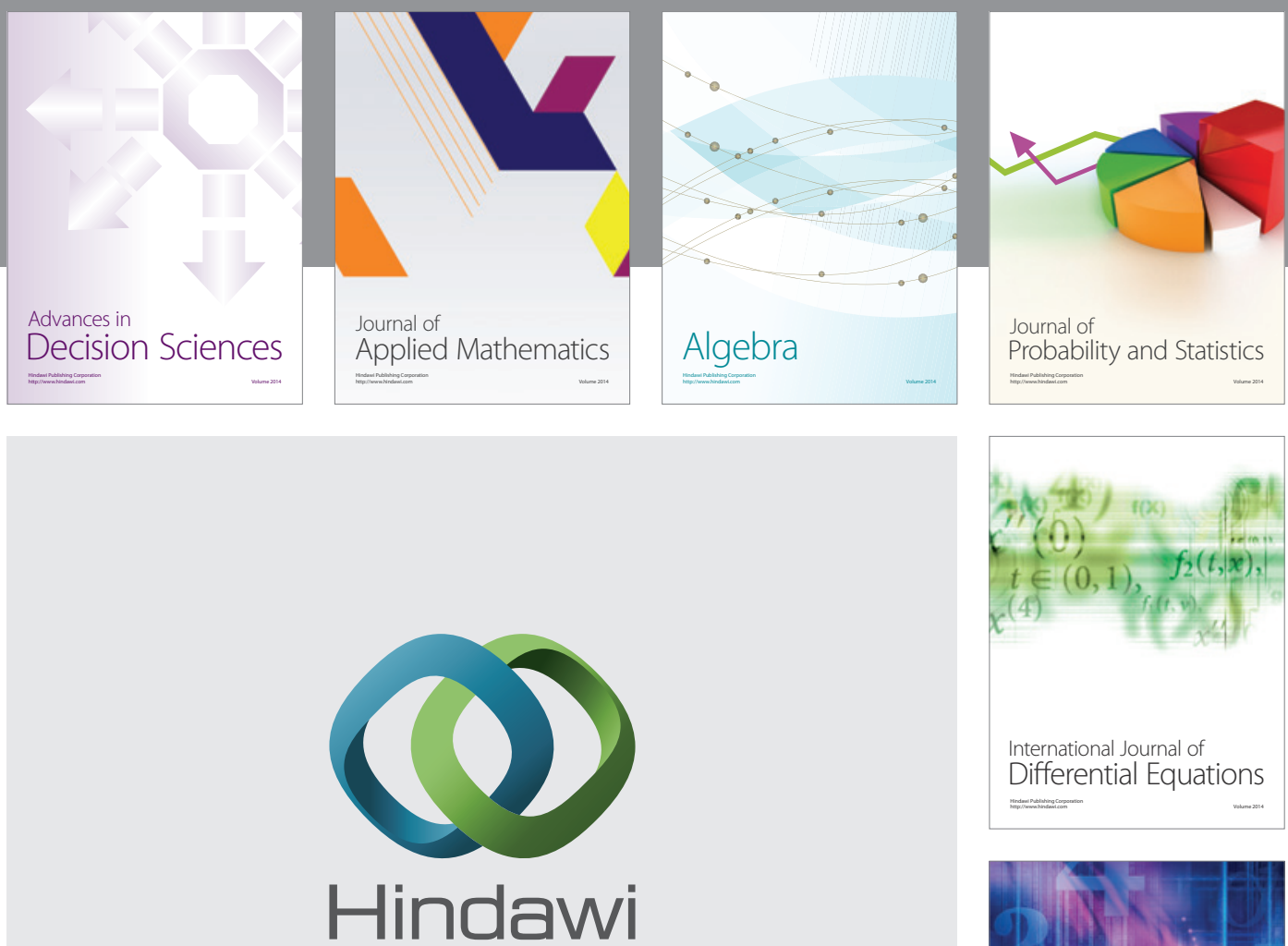

Submit your manuscripts at http://www.hindawi.com
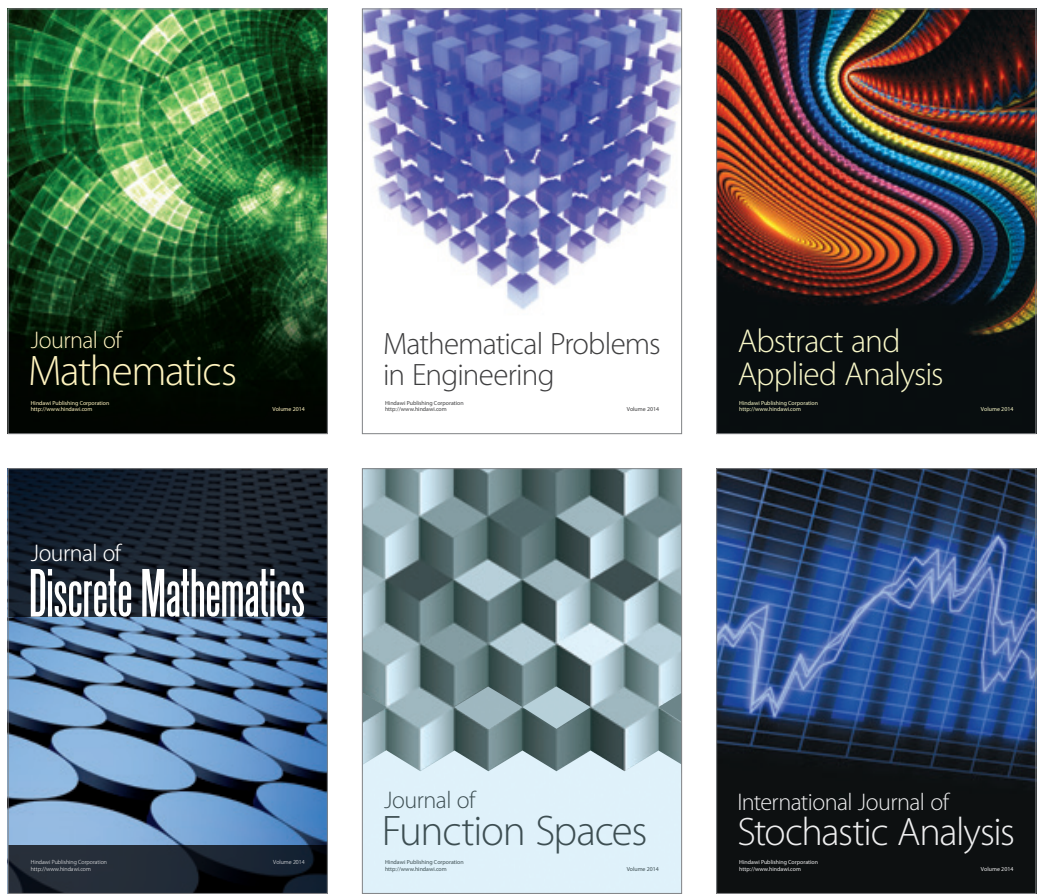

Journal of

Function Spaces

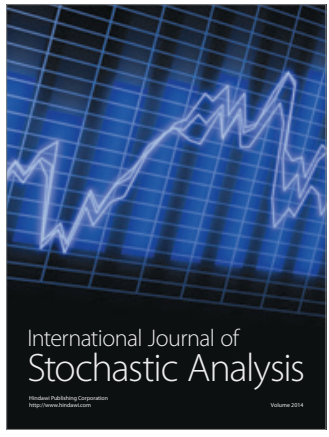

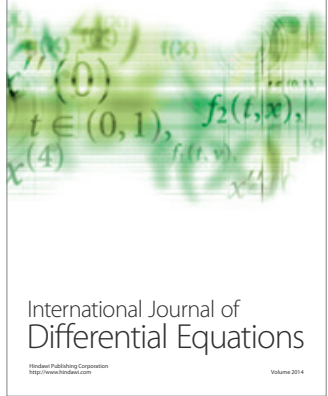
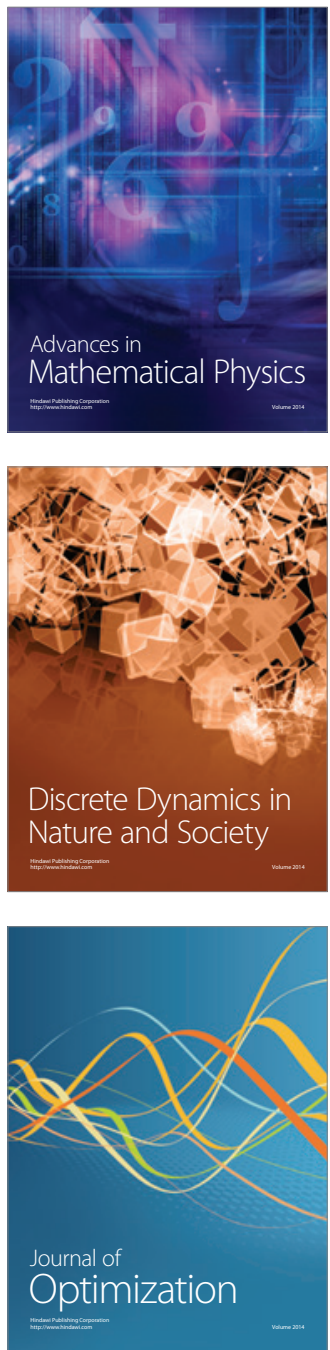